\title{
Research Activity
}

National Cancer Institute

\section{Source}

National Cancer Institute. Research Activity. NCI Thesaurus. Code C15429.

Systematic investigation into a subject in order to discover facts, establish or revise a theory, or develop a plan of action based on the facts discovered. 